\title{
Enhancing Diplomatic Effectiveness: A Common-Sense Risk Management Approach to Counter-terrorism
}

\author{
Raphael Perl *
}

Effective counter-terrorism efforts require practical and realistic risk management decisions. Since our resources are limited, clearly we should allocate them in sensible proportions and on parallel tracks in order to achieve optimal results over the long term. Unfortunately, while we are good at mobilizing resources to defend against perceived imminent threats, we often neglect or underfund other important activities, such as enhanced diplomacy, which could reduce those threats in the future.

With respect to counter-terrorism, it is difficult to make up for lost time in areas involving diplomacy. Building mutual trust and respect is an ongoing activity that cannot be rushed. Adding more people or more money later on does not shorten the time required to achieve mutual trust in the diplomatic arena. It may even delay the creation of such trust, as it indicates an inconsistency in commitment to the diplomatic process.

Therefore, it is important to provide more resources for diplomacy as soon as possible, so that when cooperative counter-terrorism efforts are needed, the groundwork is already in place to maximize effectiveness. In particular, if we are to better coordinate our intelligence efforts, it is imperative to establish lasting relationships, both bilaterally and multilaterally, to reinforce mutual trust and commitment, and to overcome any political or cultural differences that might negatively affect cooperation.

I acknowledge the dilemmas and trade-offs we face when having to choose between short-term and long-term actions. If we fail to put forth the maximum effort to stop immediate threats, there can be serious economic, political, and human consequences should a terrorist attack take place. On the other hand, if we fail to devote sufficient resources to long-term efforts directed at the mitigation of future terrorism, the threats may eventually multiply beyond our capability to defend against them. In my view, this is the greater danger.

\section{Building Prioritized and Sustainable Responses}

In terms of defending against terrorism, more is not always better, and overreacting can pose almost as great a risk as not reacting at all. Success depends upon the

\footnotetext{
Raphael Perl is the head of the OSCE Action Against Terrorism Unit, based in Vienna. A version of this paper was presented at the East-West Institute's 6th Worldwide Security Conference in Brussels in February 2009.
} 
effective use of resources, not only their quantity. Frederick the Great once said, "He who defends everything defends nothing."

Osama bin Laden, in the wake of the attacks of 11 September 2001, estimated the macro-economic cost to the world economy of the added security measures that would be implemented in response to the attacks to be in excess of USD 1 trillion. A 2004 study by the United States bipartisan Congressional Research Service supported his contention.

We have already seen a worldwide expenditure of hundreds of billions of dollars and euros on various forms of security to protect societies from terrorist threats. But much of this money has not been put to optimal use. Fearing an epidemic of terrorism, we have spent a substantial portion of our funding on largescale, expensive safeguards that are not completely effective and that require ongoing expenditures for renewal. Meanwhile, funding for diplomacy has suffered.

Terrorism has long since become a process instead of an isolated act; it is a self-sustaining global enterprise, similar to organized crime or other societal parasites. Tipping points have been reached and passed, beyond which terrorism tends to accelerate, not diminish, if left unchecked. Many aspects of terrorism, especially at the micro-level, are based on a cycle of revenge that has gone on for generations, and it will take generations more for it to abate.

Moreover, terrorism as a process has become big business, with numerous vested interests involved (and not only political interests). Arms dealers, smugglers, propagandists, criminal financiers, and other service providers have made fortunes of providing resources to terrorist organizations, and are thus strongly motivated to see terrorism expand.

Similarly, counter-terrorism has become big business. Orders for specialized machinery, guard services, sensors, unmanned aircraft, armored vehicles, and other products and services have also made fortunes for certain companies. These companies work diligently to fight terrorism, but from a financial standpoint, would they be pleased to see it disappear entirely? I do not intend to offend anyone by these comments, but counter-terrorism must be a means to an end-the necessary goal of reducing terrorism to an acceptable level - not an end or a continuously expanding process in itself.

Common sense dictates that one should not try to defend every conceivable front or point of vulnerability, but should concentrate on mitigating the potentially most destructive threats. Steps, for instance, have been taken to prevent an airliner from being used as an asymmetric weapon, and that is a key improvement. Despite the ongoing risk that a determined and sophisticated terrorist could damage or crash an airliner, we do not stop flying. Hardening essential targets is worthwhile, but massively funding defensive projects, particularly at the expense of efforts to thwart terrorist movements at their source, is a dubious approach for the long term. 


\section{Accepting the Eventuality of Losses}

In my view, sooner or later we must adopt a doctrine of "acceptable losses," taking reasonable steps to secure our vital infrastructure, while covering other eventualities through commercial insurance or government self-insurance. In short, we have to prepare for the worst to happen, while working diligently to ensure that it does not. Otherwise, we risk financial exhaustion and societal paranoia by trying in vain to protect ourselves from every conceivable attack scenario. Indeed, this exhaustion and paranoia are precisely what the terrorists seek to achieve.

We should review our priorities and free up resources to better address the most dire (albeit perhaps less likely) scenarios. A significant concern is the possible future use of weapons of mass destruction for terrorist purposes. The devastation of such an act would reach a level that would dwarf all previous terrorist attacks in the history of the world. It would seem, as a practical matter, that funding of efforts directed at preventing such a cataclysm should take precedence over nearly all other areas, but this has not been the case to date.

Everyone wants a piece of the security-spending pie, and as a result funding does not always go to the area of greatest long-term impact. We will need to make hard decisions, and accept the risk of living with lower-level losses from conventional weapons in order to redirect funding towards more important counter-terrorism activities related to weapons of mass destruction.

There is no evidence that terrorism is diminishing. On the contrary, it is decentralizing, and has become more visible if not more prevalent as a tactic. It is unrealistic to expect this trend to change until the factors that have led to its development have been mitigated. We are making some progress, but enormous work lies ahead of us. We must devise and implement strategies to preclude the indoctrination of the young into terrorist movements; to alleviate the social alienation that catalyzes terrorist recruitment; to improve living conditions in areas where hopelessness incubates hatred; to stop the cycle of revenge wherein killing begets more killing; and to facilitate nonviolent conflict resolution opportunities. Until the causative factors of terrorism unravel, or are overturned, we must learn to live with increasing threats.

In our enthusiasm for defensive measures, or even for pre-emptive action against terrorists, we should not neglect civil preparedness. For one thing, we have few means to interdict chemical or biological weapons. These weapons are in some instances relatively simple to manufacture, and have devastating consequences. A common-sense approach to dealing with attacks that we cannot prevent is to prepare for them as best we can, in order to reduce the magnitude of the damage they will cause when they inevitably occur.

We must ask ourselves in all seriousness how long we can continue to drain our economies in a futile attempt to secure everyone and everything, at all times, 
in all places. Even if it were possible to achieve such universal security - which it is not - the societal danger posed by the erosion of human rights and civil liberties from extreme security measures is real, is increasing, and has been greatly underestimated in terms of its potentially sinister implications.

\section{The Need for Enhanced Diplomacy}

So where should we put our money? This article has billed itself as dealing with common-sense solutions, and I hope my suggestions follow that theme.

My principal suggestion, as I have already implied, is to refocus attention and funding on diplomacy, with and emphasis on regional diplomacy as well as on decentralized, person-to-person diplomatic efforts.

Expanded diplomacy is a fundamental prerequisite for coordinated intelligence and coordinated action against terrorism and its causative factors.

Through improved interpersonal diplomacy, nations will find common ground for better teamwork in the multi-faceted war on terror.

Written agreements or memoranda may form a basis for joint efforts, but "connecting the dots"- the phrase used to describe a puzzle whose answer is seen only after following many linked relationships-will remain elusive until law enforcement officials and their diplomatic colleagues forge durable relationships with their counterparts in host countries.

The administrative component of diplomacy is often neglected as well, yet this is an area where a small increment of funding may bring a large positive result, while the lack of such funding may represent a significant impediment to effective international coordination. Seemingly routine diplomatic administrative support involves a huge amount of behind the scenes effort, particularly for visiting diplomatic missions. To the extent that limited staff resources delay preparations, or render them incomplete, the effectiveness of the visit, including the coordination of intelligence, may be reduced.

Expanded diplomacy is a common-sense approach to enhancing present and future counter-terror efforts at many levels and on many fronts. We should encourage relationships on a personal level, including constructive diplomatic engagement, both with friends and with others who are not yet our friends. Our diplomats should be given sufficient time and support to help sow the seeds of mutual trust, respect and understanding, despite political and cultural differences.

There will certainly be many areas of bitter disagreement, where the line between a terrorist and a freedom fighter is blurred. We should set them aside where possible, and work towards some degree of cooperation, however limited it may be, toward common, realistic, achievable goals.

It is important to keep in mind that not all countries, national groups, or ideologies condemn terrorism. Some may regard it as a legitimate tactic. Peace is a fragile concept. The saying that it is necessary to "win hearts and minds" has only 
become cliché because it has been done poorly in the past. Nevertheless, it is right on target. Ideological engagement, debate, and discussion are integral parts of public diplomacy. If we wish to mitigate terrorism, we must understand it.

\section{Reallocating Resources to Reinvigorate Diplomacy}

Unfortunately, despite the burgeoning expenditures for security measures, funding for diplomacy has not kept pace with the expanding scope of this work that is so critical for success in the long run.

Some governments presume that improved global communications allow more centralized management of foreign policy directly from foreign ministries, rather than via embassies. I believe that decentralized diplomacy is actually far more important in the modern world, particularly in the area of counter-terrorism.

Diplomats from all nations are well-trained, loyal, and service-oriented. Their common problem is a lack of time and resources, not a deficit of skill or dedication.

In recent years, many countries have significantly curtailed or retrenched their diplomatic activities. For example, the formerly independent U.S. Information Service, the public diplomacy arm of the U.S. government, was merged several years ago with the U.S. Department of State. Some might argue that this consolidation brings economies of scale, the savings from which lead to improved coordination, but this remains to be seen, particularly with respect to public diplomacy efforts to mitigate the roots of terrorism.

Workloads at embassies abroad keep growing, while staff numbers usually do not keep pace, or may even be cut. The result is a gradual erosion of quality and effectiveness of the diplomatic work that is accomplished. Among the first areas to be scrutinized for cuts during a budget review are:

- Representational expenses (other than for top officials) for establishing personal contacts with counterparts in other embassies and in the local foreign ministry

- Cultural and educational exchanges, as well as other aspects of public diplomacy, such as multicultural libraries, radio stations, and language courses

- Administrative expenses, such as logistics, support costs, routine scheduled maintenance of building infrastructure and vehicles, and other services whose curtailment could affect the efficiency of diplomats in their daily work

- Frequency and degree of coordination with local law enforcement officials in consular areas. 
Person-to-person diplomacy is conducted with great success on a limited scale by certain senior embassy personnel, but this is not enough. We should expand and encourage contacts with host country diplomats, and other officials and personnel by all levels and categories of officers.

This cannot be accomplished by simply assigning extra duties. We must staff embassies at a level corresponding to the actual workload, rather than simply assigning more work to people whose schedules are already full. It takes time and regular contact in order for interpersonal diplomatic relationships to form. In most countries this does not happen in just a few weeks, or even after several months.

\section{Diplomacy as an Insurance Policy}

Diplomacy, like security, is expensive, but it should be regarded as a type of "insurance policy," which one hopes will reduce the risk of terrorism through prevention or mitigation. It is through diplomacy that we can strive to mitigate terror in the long run. If successful, this approach leverages the money spent on diplomacy by a huge multiplier in terms of avoided security costs.

As was mentioned above, an effective investment is regional diplomacy, particularly through the medium of regional organizations, where a number of institutions and governmental bodies have specific roles as regional facilitators and coordinators, in a variety of areas of interest. Terrorism is a common concern to many if not all regional institutions, such as the Organization for Security and Cooperation in Europe (OSCE), the European Union, the Council of Europe, the Organization of American States (OAS), the Asia Pacific Economic Cooperation (APEC) group, to mention only a few.

The OSCE plays an important role in promoting cooperation and capacitybuilding among its fifty-six participating states in counter-terrorism and other related areas. It offers a weekly forum for countries to present their messages to foreign ambassadors and other senior officials. The value of such an opportunity must not be underestimated; indeed, it should be fully exploited. Moreover, as the experience of the OSCE has shown, cooperation between regional organizations and global bodies adds further value by helping to leverage limited resources and amplify important political messages.

In addition, there are other entities - particularly non-profit, non-governmental organizations - that also focus on diplomacy, multi-cultural understanding, and similar aspects of national and international cooperation. These contribute effectively to diplomacy, and through it, to counter-terrorism and other coordinated efforts.

With more diplomacy and improved interpersonal engagement, we could look forward to obtaining more and better data concerning the factors that contribute to terrorism from sources such as focus groups, attitude and opinion surveys, econometrics, descriptive and predictive statistics of societal trends, and similar meas- 
urement tools. There has been a shortage of such data in the past, and without reliable, valid data, it is difficult to formulate effective counter-terror strategies.

We are likely to witness terrible tragedies in the future from acts of terrorism. We will not be able to prevent them all. To the extent that we can mitigate the process of terrorism by diverting more funds toward diplomatic efforts, and by more realistically accepting certain levels of risk in other areas, we will achieve better coordination of intelligence and more effective regional and international cooperation in dealing with crises. Through more effective diplomacy, our children and grandchildren may live in a world with substantially less terror than we currently face. 